\title{
HISTome2: a database of histone proteins, modifiers for multiple organisms and epidrugs
}

Sanket G. Shah ${ }^{1,3 \dagger}$, Tushar Mandloi ${ }^{2 \dagger}$, Pooja Kunte ${ }^{1,4 \dagger}$, Abhiram Natu ${ }^{1,3 \dagger}$, Mudasir Rashid ${ }^{1,3 \dagger}$, Divya Reddy ${ }^{1,3,5}$, Nikhil Gadewal $2^{*^{*}}$ and Sanjay Gupta ${ }^{1,3^{*}}$

\begin{abstract}
Background: Epigenetics research is progressing in basic, pre-clinical and clinical studies using various model systems. Hence, updating the knowledge and integration of biological data emerging from in silico, in vitro and in vivo studies for different epigenetic factors is essential. Moreover, new drugs are being discovered which target various epigenetic proteins, tested in pre-clinical studies, clinical trials and approved by the FDA. It brings distinct challenges as well as opportunities to update the existing Hlstome database for implementing and applying enormous data for biomedical research.

Results: HISTome2 focuses on the sub-classification of histone proteins as variants and isoforms, post-translational modifications (PTMs) and modifying enzymes for humans (Homo sapiens), rat (Rattus norvegicus) and mouse (Mus musculus) on one interface for integrative analysis. It contains 232, 267 and 350 entries for histone proteins (noncanonical/variants and canonical/isoforms), PTMs and modifying enzymes respectively for human, rat, and mouse. Around 200 EpiDrugs for various classes of epigenetic modifiers, their clinical trial status, and pharmacological relevance have been provided in HISTome2. The additional features like 'Clustal omega' for multiple sequence alignment, link to 'FireBrowse' to visualize TCGA expression data and 'TargetScanHuman' for miRNA targets have been included in the database.

Conclusion: The information for multiple organisms and EpiDrugs on a common platform will accelerate the understanding and future development of drugs. Overall, HISTome2 has significantly increased the extent and diversity of its content which will serve as a 'knowledge Infobase' for biologists, pharmacologists, and clinicians. HISTome2: The HISTone Infobase is freely available on http://www.actrec.gov.in/histome2/.
\end{abstract}

Keywords: Histones, PTMs, Enzymes, Epidrugs

*Correspondence: ngadewal@actrec.gov.in; sgupta@actrec.gov.in

${ }^{\dagger}$ Sanket G. Shah, Tushar Mandloi, Pooja Kunte denotes equal first author contribution

${ }^{\dagger}$ Abhiram Natu and Mudasir Rashid denotes equal second author contribution

1 Epigenetics and Chromatin Biology Group, Gupta Laboratory, Cancer Research Institute, Advanced Centre for Treatment, Research and Education in Cancer, Tata Memorial Centre, Kharghar, Navi Mumbai, MH 410210, India

${ }^{2}$ Bioinformatics Centre, Advanced Centre for Treatment, Research and Education in Cancer, Tata Memorial Centre, Kharghar, Navi Mumbai, $\mathrm{MH} 410210$, India

Full list of author information is available at the end of the article

\section{Background}

The nucleosome is a fundamental unit of chromatin that plays an essential role in governing biological processes like gene expression, gene regulation, and DNA repair. Each nucleosome consists of $\sim 147$ base pairs of DNA wrapped around an octamer of histone proteinsa tetramer of $\mathrm{H} 3 / \mathrm{H} 4$ and two dimers of $\mathrm{H} 2 \mathrm{~A} / \mathrm{H} 2 \mathrm{~B}$ [1]. Histones are usually categorized into 'canonical' and 'non-canonical'. Canonical histones, also defined as histone isoforms, are located in clusters at a gene level and are exclusively expressed in the S-phase of the cell cycle with 3' stem-loop organization. Whereas

c) The Author(s) 2020. This article is licensed under a Creative Commons Attribution 4.0 International License, which permits use, sharing, adaptation, distribution and reproduction in any medium or format, as long as you give appropriate credit to the original author(s) and the source, provide a link to the Creative Commons licence, and indicate if changes were made. The images or other third party material in this article are included in the article's Creative Commons licence, unless indicated otherwise in a credit line to the material. If material is not included in the article's Creative Commons licence and your intended use is not permitted by statutory regulation or exceeds the permitted use, you will need to obtain permission directly from the copyright holder. To view a copy of this licence, visit http://creativeco mmons.org/licenses/by/4.0/. The Creative Commons Public Domain Dedication waiver (http://creativecommons.org/publicdomain/ zero/1.0/) applies to the data made available in this article, unless otherwise stated in a credit line to the data. 
non-canonical histones or histone variants are solitary genes, expressed in a replication-independent manner throughout the cell cycle with poly-A tail [2]. The isoforms vary among themselves only by a few amino acids, whereas variants significantly differ from canonical histones as well as among each other.

Structural shifts between euchromatin and heterochromatin take place by reversible incorporation of histone variants in the nucleosomes with site-specific post-translational modifications. Earlier studies have shown that the replacement of a canonical histone by a variant is a dynamic process and account for the complexity contributing to the cell fate and genome plasticity [3-6]. Recently, histone isoforms are also emerging as an important player in the field of chromatin biology and have shown to be functionally non-redundant [7]. Growing evidence suggests that aberrant regulation of gene expression through the incorporation of histone isoforms and variants, and their site-specific posttranslational modifications are strongly associated with different human diseases like cancer, diabetes, adiposity, auto-immune diseases, Alzheimer's disease [8-11]. The macroH2A levels were found to be decreased in lung cancer [12]. H2A.Z was also found to be associated with expression of cell proliferation and differentiation related genes $[13,14]$. Further, the overexpression of H2A.Z.2 was found to be associated with poor survival in melanoma [15]. H3 variant CENP-A expression is also reported to increase in breast and colon cancer [16]. Further, the differential expression of histonemodifying enzymes is reported in various human diseases for site-specific histone modifications [17]. High levels of H4K5Ac, H3K27Ac, H3K18Ac, H4K8Ac are associated with lung and ovarian cancer $[18,19]$. HATs like CREBBP (CREB binding protein) and EP300 were found to be over-expressed in the colon and small cell lung cancers $[20,21]$. In parallel, HDAC 1,2 and 3 were also reported to over-express in gastric, lung, breast and hepatocellular cancers [22]. Histone methyl transferase, EZH2 along with site-specific methylation, H3K27me3 were found to be upregulated in breast and prostate cancer [23]. Different kinases and ubiquitinases were also reported to be altered in cancer [24, 25]. In the last 15 years, a different class of drugs known as epidrugs has been developed that target the histonemodifying enzymes. Epidrugs have shown promising results in pre-clinical models as well as in clinical trials. In particular, new and specific DNMT and HDAC inhibitors were screened in cell lines and pre-clinical animal models of several cancers, such as breast, skin, colorectal and liver. The DNMT and HDAC inhibitors are also approved by the FDA for use in clinics against cancer and other diseases [26].
In the past decade, several databases related to different aspects of chromatin biology were developed due to the importance of histone proteins in the regulation of DNA-mediated cellular processes. The databases such as Human Histone Modification Database (HHMD) gives information about experimentally identified human histone PTMs [27], ChromDB provides details about histone modifications on Saccharomyces cerevisiae [28], Histone Systematic Mutation Database (HistoneHits) contains relevant data about histone mutants in yeast [29] and Histone Database aims to focus on histone structures and sequences in many species [30]. Also, the human epigenetic drug database (HEDD) focuses on epigenetic drugs obtained from experiments and curated data [31]. The earlier version of the HIstome database provides information about histone proteins, histone PTMs and its modifiers in humans [32]. The key importance of histones and associated functional proteins along with ever-growing information in multiple cellular functions, organisms, and diseases is necessary to update the current database, HIstome: The Histone Infobase.

In light of the above needs, the HIstome database is updated to HISTome2: The Histone Infobase, which is available at http://www.actrec.gov.in/histome2/. The new Infobase has been created, which covers information on histone proteins, variants, and isoforms separately; their sites of modifications and modifying enzymes from mammalian systems such as Homo sapiens, Rattus norvegicus, and Mus musculus. Further, to visualize RNA expression of human epigenetic enzymes and histone proteins in cancer, a link to TCGA FireBrowse has been included [33]. A list of all putative miRNAs for all human entries has been provided via TargetScan [34]. The literature for all the entries is made available by connecting each entry with PubMed. Similarly, our new version of the database introduces detailed information about epidrugs and their ongoing experimental, pre-clinical and clinical studies. The EpiDrug database entries have been categorized by the classes of histone- or nucleic acid-modifying enzymes with their known inhibitors like HATi, HDACi, DNMTi, etc. Further, to enhance the utility of database for the scientific community, new features such as multiple sequence alignment (MSA), histone isoform dimer stability prediction by energy minimization, and advanced search have been integrated to extract query-based information. MSA will help in understanding the sequence similarities and conservation of histone proteins across species. Further, the difference in energy minimization of highly conserved histone dimers might get reflected on nucleosome stability.

Overall, the updated version of the histone database has significantly increased the extent, diversity of its content and thus assisting in the search and comparative 
interpretation of the multifactorial parameters in the field of histone biology. The comprehensive information available on the database will serve as a useful "knowledge base" for fundamental researchers, clinical scientists, pharmacologists in understanding the histone biology and their potential importance in epidrug discovery through its ability to connect chemistry, biology, and informatics.

\section{Results: updates and new features Home page for an integrative search for multiple organisms, EpiDrug, and tools}

The new version, HISTome2, has been designed to integrate the information of human, mouse, rat and EpiDrug database by a user-friendly interface. The database home page consists of two bars viz. navigation and menu bar. The schema of database is mentioned in construction part of methods section (Fig. 1). The navigation bar helps the user to navigate to the individual databases for human, mouse, rat, and EpiDrug. The menu bar helps the user to retrieve information about the variants, isoforms, PTMs, writers, and erasers for all organisms. For example, upon bringing the cursor over variants, isoforms, writers, and erasers, a drop-down menu will appear whereby with a single click, the user can retrieve the information of all the three organisms at a time. However, for PTMs, clicking on respective modifications, common as well as a unique site of modification will be displayed. Also, the video tutorial has been included under 'how to use' for easy navigation through the database.

\section{New inclusion and updation of the entries}

In the updated version, the information for the histone proteins is subcategorized as canonical and non-canonical. These histone proteins, their PTMs and modifying enzymes for human, mouse, and rat are included in the MySQL database. An earlier version of the HIstome database contained information of 55 histone proteins, 106 PTMs, and 152 modifying enzymes only for human database [32]. In the updated version HISTome2, the histone proteins for humans contain 97 entries with a sub-classification of 33 variants (non-canonical) and 64 isoforms (canonical), 114 distinct sites of histone modifications, and 161 modifying enzymes. The new information for routinely used pre-clinical model systems, rat and mouse is included in the HISTome2. Rat database carries 48 entries of histone proteins with subdivision of 21 variants (non-canonical), and 27 isoforms (canonical), 61 distinct sites of their modifications and 89 modifying enzymes. Mouse database has 87 entries of histone proteins which are sub-categorized into 26 variants (non-canonical) and 61 isoforms (canonical),

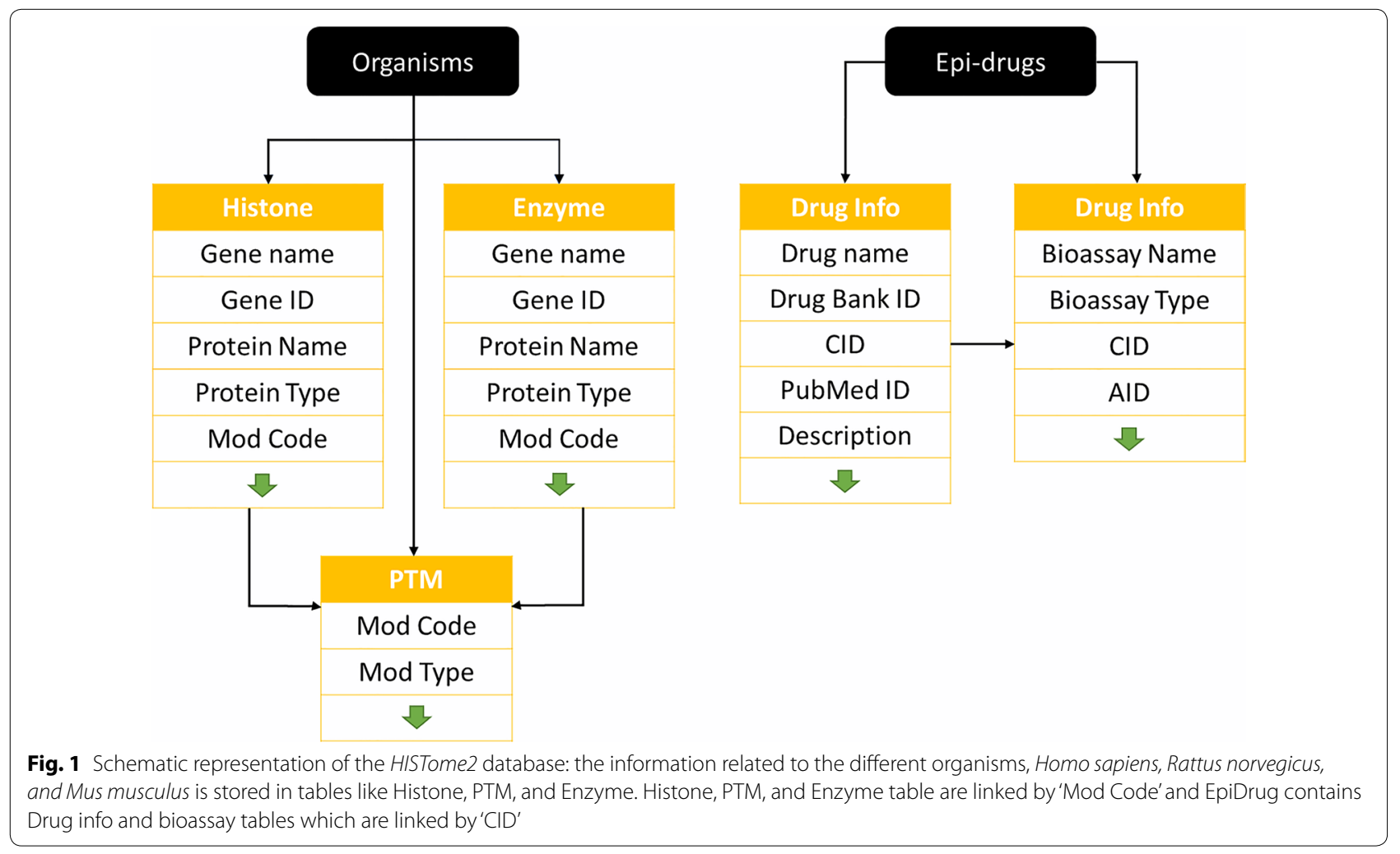


92 distinct sites of their modifications and 100 modifying enzymes. To each entry of histone proteins and modifying enzymes in the database, a 'detailed information page' is developed, which gives hyperlinks to external databases for further information (Fig. 2). Further, to gain the real-time updated references of these entries, a link to PubMed is provided with pre-embedded keywords.

The differential gene expression of histone variants, isoforms, and epigenetic modifiers has been reported in various pathophysiological conditions like cancer. The information related to altered expression of histone variants, isoforms, and epigenetic modifiers in human

\section{Histone $\mathrm{H} 3.2$}

Histone H3.2 isoform expression is replication dependent and it mediates transcriptional repression.

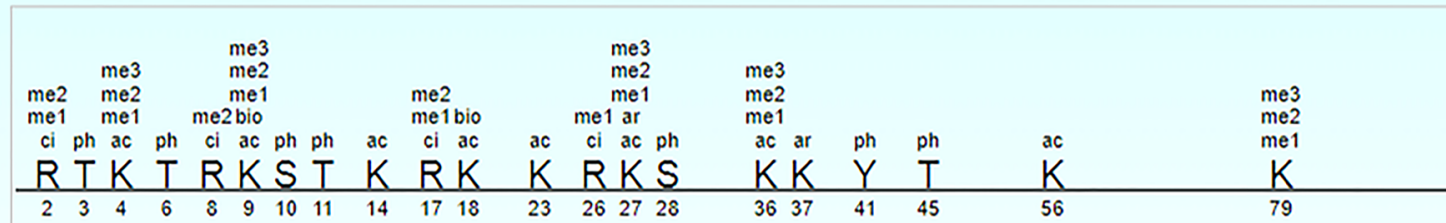

Click on PTM for further information

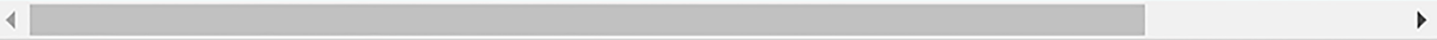

\section{Download table as Excel}

\begin{tabular}{|c|c|c|c|}
\hline \multicolumn{4}{|c|}{ Histone H3.2 infosheet } \\
\hline $\begin{array}{l}\text { UniprotKB } \\
\text { Accession }\end{array}$ & \multicolumn{3}{|c|}{$\underline{Q 71 D I 3}$} \\
\hline Alternative Name(s) & \multicolumn{3}{|c|}{ Histone $\mathrm{H} 3 / \mathrm{m}$, Histone $\mathrm{H} 3 / 0$} \\
\hline $\begin{array}{l}\text { No. of coding } \\
\text { genes }\end{array}$ & \multicolumn{3}{|c|}{ Histone $\mathrm{H} 3.2$ is coded by following 3 non-allelic gene/s } \\
\hline Gene name & histone cluster $2, \mathrm{H} 3 \mathrm{c}$ & histone cluster $2, \mathrm{H} 3 \mathrm{a}$ & histone cluster $2, \mathrm{H} 3 \mathrm{~d}$ \\
\hline Gene symbol & $\mathrm{HIST} 2 \mathrm{H} 3 \mathrm{C}$ & $\mathrm{HIST} 2 \mathrm{H} 3 \mathrm{~A}$ & $\mathrm{HIST} 2 \mathrm{H} 3 \mathrm{D}$ \\
\hline $\begin{array}{l}\text { Promoter region } \\
(-700 \text { TSS }+300)\end{array}$ & Get sequence & Get sequence & Get sequence \\
\hline Previous Symbol/s & H3F2, H3FM & & \\
\hline Aliases & MGC9629, H3/m, H3, H3.2, H3/M & $\mathrm{H} 3 / \mathrm{n}, \mathrm{H} 3 / \mathrm{o}$ & \\
\hline GenelD & $\underline{126961}$ & $\underline{333932}$ & $\underline{653604}$ \\
\hline HGNC & $\underline{20503}$ & $\underline{20505}$ & $\underline{25311}$ \\
\hline OMIM & NULL & NULL & $\underline{\text { NULL }}$ \\
\hline RefSeq mRNA & NM 021059.2 & NM_001005464.2 & NM 001123375.2 \\
\hline RefSeq Protein & NP_066403.2 & NP_001005464.1 & NP_001116847.1 \\
\hline $\begin{array}{l}\text { TCGA Gene } \\
\text { Expression } \\
\text { (Firebrowse) }\end{array}$ & HIST2H3C & HIST2H3A & $\underline{\mathrm{HIST}} 2 \mathrm{H} 3 \mathrm{D}$ \\
\hline mir Targets & HIST2H3C & HIST2H3A & $\underline{\mathrm{HIST} 2 \mathrm{H} 3 \mathrm{D}}$ \\
\hline Pubmed & For more Information & For more Information & For more Information \\
\hline
\end{tabular}

Fig. 2 Representative image of histone entry page for Human: The individual entry has brief write-up for biological role, site-specific modifications and different content which are divided into uniport ID, synonym, number of coding genes, gene name, gene symbol, promoter region, gene ID, HGNC, Refseq mRNA, Refseq protein, TCGA expression and mIR targets for a specific histone 
is available on TCGA (The Cancer Genome Atlas) databases [35]. Therefore, the HISTome2 database provides a link to TCGA FireBrowse from which expression profiles of different histones in normal and cancer of various tissue types in humans can be extracted [33]. The expression of histone genes and modifying enzymes is also regulated by microRNAs; therefore, a link to the TargetScan database is provided to extract the probable
microRNAs that can regulate expression of specific target genes [34].

The new inclusion, EpiDrug database, highlights the different types of inhibitors based on the chromatinmodifying enzymes that either 'write' or 'erase' the functional groups. The individual category summarizes chemical molecules and potential drugs that are either approved by the FDA or are currently being used in in vitro or pre-clinical experimental studies. A total of

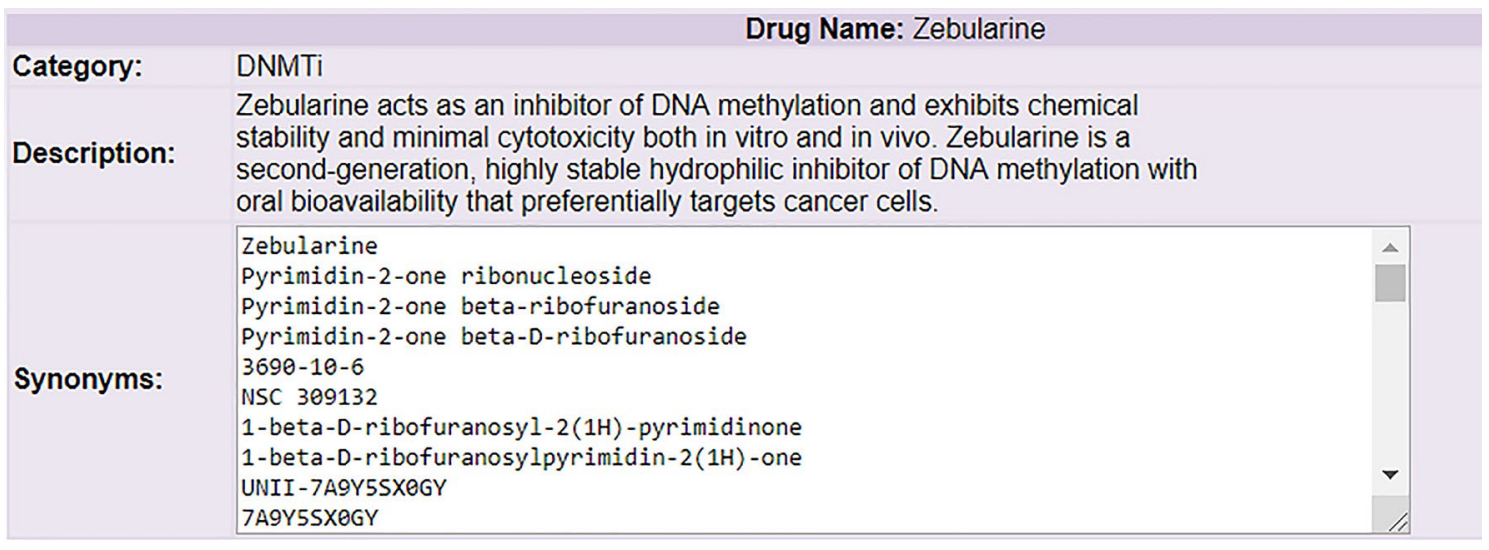

Structural Information: Zebularine

2D Image:

Structure 2D:

Molecular Formula:

Molecular Weight:

IUPAC name:

InChl:

SMILES:
Download

$\mathrm{C} 9 \mathrm{H} 12 \mathrm{~N} 2 \mathrm{O} 5$

$228.204 \mathrm{~g} / \mathrm{mol}$

1-[(2R,3R,4S,5R)-3,4-dihydroxy-5-(hydroxymethyl)oxolan-2-yl]pyrimidin-2-one

InChl=1S/C9H12N2O5/c12-4-5-6(13)7(14)8(16-5)11-3-1-2-10-9(11)15/h1-3,5-8,12-14H $4 \mathrm{H} 2 / \mathrm{t} 5-, 6-, 7-, 8-/ \mathrm{m} 1 / \mathrm{s} 1$

$\mathrm{C} 1=\mathrm{CN}(\mathrm{C}(=\mathrm{O}) \mathrm{N}=\mathrm{C} 1) \mathrm{C} 2 \mathrm{C}(\mathrm{C}(\mathrm{C}(\mathrm{O} 2) \mathrm{CO}) \mathrm{O}) \mathrm{O}$

\section{FDA status:}

FDA_UNII:

Clinical Trials :
Clinical Information: Zebularine

\section{Experimental}

7A9Y5SX0GY

Not Recruited Clinical Trials

Fig. 3 Representative image of epidrug 'Zebularine', a DNA methyltransferase inhibitor: the entry of 'Zebularine' epidrug is divided into multiple pieces of information like basic, structural, clinical, bioassay and references in the database 
200 molecules have been identified by searching PubMed and pharmaceutical websites (https://www.medchemexp ress.com/Pathways/Epigenetics.html) which are categorized into 12 different types. The individual entries of these molecules have information regarding their structure, chemistry, bioassay, and current phase trial status with a link to the ClinicalTrial.gov website for detailed information (Fig. 3). Further, the database also provides information about basic molecular properties like weight, formula, etc. for each drug. Three different chemical descriptors have been provided for each compound: (i) International Union of Pure and Applied Chemistry (IUPAC), (ii) Canonical Simplified Molecular-Input LineEntry System (SMILES) [36-38], and (iii) IUPAC International Chemical Identifier (InChI) [39-41]. Also, the bioassay information is linked to the PubChem Bioassay website using the ID (AID) of each assay for providing data related to pharmacology, patents, and bioactivities. Further, individual drugs have been linked to the different databases like ChEMBL [42], ZINC DB [43], Human Metabolome DB [44], LiverTox [45] and Small Molecule Pathway Database [46] to give added information about their structures, toxicity and the biological impact on different tissues of human body after consumption of the drug.

\section{Sequence alignment of histone isoforms and variants}

Multiple Sequence Alignment helps in aligning different proteins based on sequence similarities. The sequence alignment page displays a list of various histone variants and isoforms in human, rat, and mouse. The user can select single or multiple histone proteins from a single organism using the check-boxes or can compare protein sequences across the three species by selecting specific variants or isoform among the three organisms. For example, the output of multiple sequence alignment of histone $\mathrm{H} 3$ isoforms from human, mouse, and rat shows the favorable substitution position 87 (in blue); position 90 and 96 (in black) shows the unconserved region and identical amino acids are in red (Fig. 4). In continuation, the WebLogo indicates the overall height for the conserved amino acids, whereas the height at 87, 90 and 96 is adjusted based on the relative frequency of occurrence in an alignment. Histone isoforms within species and across species are quite similar. They differ by a few amino acids (1-3) within species. Therefore, MSA will provide information about the conservation of protein within and across species. The presence of specific amino acids in a protein sequence gives rise to specific secondary or tertiary structures. Even a single unfavorable amino acid substitution can disrupt the stability of the protein structure. Hence, studying regions of favorable substitutions, mutations, and conservations in the amino acid sequence become necessary to understand its importance in determining the protein's structural integrity and its functional impact. The difference in the amino acid sequence could be the possible reason for structural and functional variability among the different histone isoforms. Also, based on the algorithm, one can predict the phylogenetic distance between the species using a given histone protein sequence. Therefore, with the help of the sequence alignment tool, researchers can study MSA of different histone orthologs from the given three organisms and explore their evolutionary relationship. The tool provides a pictorial representation of regions of conservation and substitutions, which could help researchers in studying variation among histones.

\section{Histone dimer stability prediction for histone H2A and H2B isoforms}

In nucleosome organization, the wrapping of DNA with histone dimer of $\mathrm{H} 2 \mathrm{~A} / \mathrm{H} 2 \mathrm{~B}$ and tetramer of $\mathrm{H} 3 / \mathrm{H} 4$ plays a vital role in the formation of a stable nucleosome. Earlier studies have shown that differential incorporation of histone variants leads to changes in nucleosomal architecture [47]. The histone variants are not included in the present versions of the database for calculating energy minimization, as literature is available about their role in the stability of nucleosomes [47]. Histone H2A and H2B have multiple isoforms, $\mathrm{H} 3$ has two isoforms and $\mathrm{H} 4$ has none in humans [7]. The histone isoforms and their role in nucleosomal stability have not been studied in-depth; therefore, in the updated version, information related to the potential energy of $\mathrm{H} 2 \mathrm{~A} / \mathrm{H} 2 \mathrm{~B}$ isoform dimers is included to predict the differences in the stability of nucleosome. The outputs are represented as a heatmap of histone $\mathrm{H} 2 \mathrm{~A}-\mathrm{H} 2 \mathrm{~B}$ dimer with their potential energy. Earlier studies from our lab have shown that the nucleosomal stability of $\mathrm{H} 2 \mathrm{~A} 1 \mathrm{H} / \mathrm{H} 2 \mathrm{~B}$ was different compared to H2A.2/H2B, in silico as well as in vitro [48, 49]. At the dimer level also the stability was different for both the isoforms.

\section{Conclusion and future strategies}

HISTome 2 is a freely available web-based resource that provides comprehensive information on histone isoforms, variants, histone PTMs, modifying enzymes, and epidrugs against 'erasers' and 'writers' and their pre-clinical as well as clinical trial status on a common platform. Histone isoforms and variants can also be compared across multiple organisms to understand their evolution/ orthologs in other species. The common platform provides scientists to explore histone entries between and across organisms, their expression in cancer and potential miR targets. The inclusion of epidrugs against epigenetic modifiers on a common platform with histone-modifying 


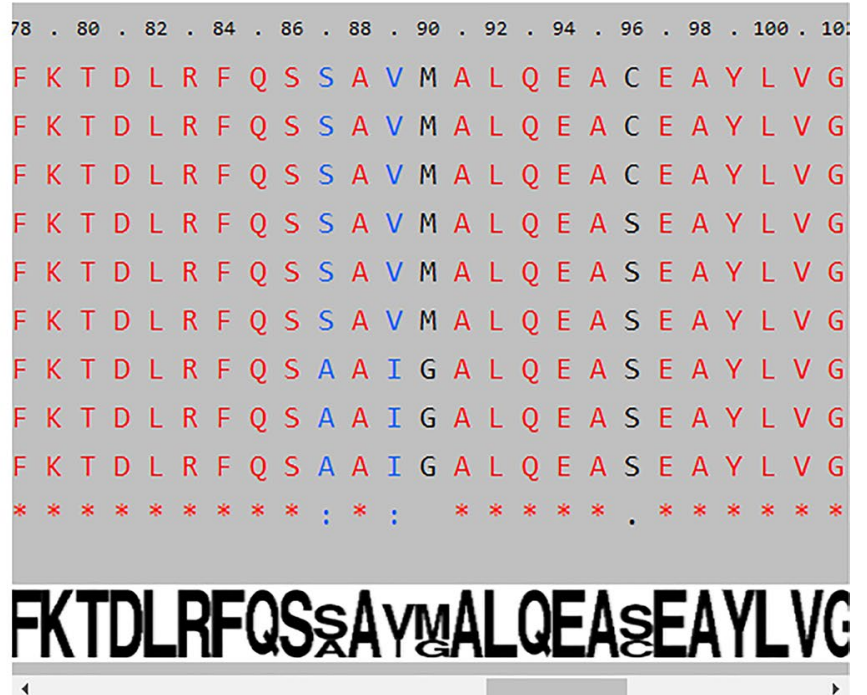

Fig. 4 Representative image of sequence alignment of histone H3 proteins from human, mouse, and rat: the amino acids in 'red' color indicate identical residues in all proteins, 'blue' indicate amino acids substituted with similar properties among the queried proteins, while 'black' indicate amino acids with different properties among proteins. A'WebLogo' is placed parallel below the alignment to highlight the relative frequency of occurrence

enzymes will significantly enhance the possibility of successful planning of the pre-clinical and clinical research. The entries are connected with a peripheral link to different freely available databases to retrieve detailed and real-time updated information. The information on all these epigenetic factors in other species is also emerging rapidly. Thus, we intend to update the current database along with the integration of information for other eukaryotic organisms like yeast, C. elegans, and zebrafish in the future.

\section{Methods}

\section{Constructions and content of HISTome2} Home page for integrative search

The interface and visualization of the HISTome 2 are developed using XHTML, JavaScript, PHP, and MySQL. The webpages of the HISTome 2 are dynamically loaded using AJAX and JavaScript functions, which are processed by PHP to present the data from MySQL. The schema of the database and connections between the tables is shown in Fig. 1. The information on human, mouse and rat database is stored in histone, PTM and enzyme tables which has a common schema to perform an integrative search on single keyword input. Whereas, the data on epidrugs are stored in drug info and bioassay tables which are connected by primary key 'CID'. The data are stored in an MySQL relational database with 3-tier architecture viz. database, user and application tiers. The database tier contains the data stored in the form of tables; the user tier provides access to a user interface. The data in the application tier are accessed by Clustal Omega [50] and WebLogo [51].

\section{Data mining and links to peripheral resources}

In HISTome2, the information on histone proteins, PTMs and modifying enzymes in human, rat and mouse was manually curated from NCBI, uniport, gene-cards, histone DB2.0, and Talbert et al. [52-56]. The methodology for data mining for 'detailed information page' was modified from the earlier version (HIstome) because for chromosomal location, URL was no longer available and the Unigene database will be discontinued shortly. Therefore, UniProt accession ID was provided for all the entries as it is the most comprehensive protein database which provides detailed functional information for the proteins. Further, gene-related information such as name, symbol, and GeneID was acquired from HGNC [57], RGD [58], and MGI [59] for human, rat, and mouse, respectively. Gene, transcript, and protein-related information were obtained from NCBI, while EC number was fetched from EC-PDB [60]. Promoter sequences for different histones and modifying enzymes were obtained from EPD [61]. The PubMed link was provided to each entry with preembedded aliases in keywords to fetch updated information and to exclude non-specific searches. However, if the PubMed search did not display any literature, a specific PMID reference link is provided. An additional hyperlink was provided to all the entries for humans to retrieve TCGA mRNA expression from FireBrowse [33] and mIR targets from TargetScan [34]. 
A new component in the database, EpiDrug is added to highlight its importance in the field of epigenetics and potential in the treatment of different diseases. The information on the epidrugs was retrieved from PubMed, PubMed Central, and Google scholar, using different searches related to DNA methylation and histone-modifying enzymes like DNA methyltransferase inhibitors, histone acetyltransferase inhibitors, histone deacetylase inhibitors, histone methyltransferase inhibitors, histone acetyltransferase inhibitors, histone demethylase inhibitors, inhibitors of proteins binding to methylated histones, protein arginine deiminases inhibitors, poly (ADP-ribose) polymerase inhibitors, inhibitors of bromodomain (BRD) and extra-terminal domain (BET) family of proteins, and inhibitors of ubiquitinases and deubiquitinases. The information on synonyms, molecular formula, molecular weight, IUPAC, InChl, smiles, and 2D structures of epidrugs are obtained from the PubChem compound database [62]. The list of biological assays that have been performed on these chemical compounds to determine the chemical toxicity and bioactivity is acquired from the PubChem BioAssay database [2]. The information on the FDA status and ongoing clinical trials is fetched from ClinicalTrial.gov [63].

\section{Data repository and analysis tools Sequence alignment}

To elucidate the role of conserved amino acids in different types of histones isoforms and variants within an organism and across the three organisms, the sequence alignment tool is introduced in HISTome2. The selected protein sequences are processed using AJAX script and serve as an input to Clustal Omega for sequence alignment and return the complete output as a string [50]. Clustal Omega was run with default parameters such as gap penalty 10, gap extension penalty 0.20 and GONNET protein weight matrix. The alignment output file is handled using PHP scripts by displaying the alignment in a single-line scrollable window with the number assigned to amino acids in an alignment. The conserved residues (identities) in an alignment are marked with an asterisk in red color and while conservative substitution (similar) residues displayed with a colon in blue color. The same alignment file (output of clustal omega) is given as an input to the WebLogo tool to generate WebLogo image, which is aligned with the Clustal Omega output using javaScript [51].

\section{Histone dimer stability prediction for histone $\mathrm{H} 2 \mathrm{~A}$ and $\mathrm{H} 2 \mathrm{~B}$ isoforms}

The binding affinity between histone dimers dictates the stability of the nucleosome [49]. The PDB ID: 2CV5 was taken as a template structure for building histone $\mathrm{H} 2 \mathrm{~A}$ and $\mathrm{H} 2 \mathrm{~B}$ isoforms dimer of human, mouse, and rat using Discovery studio visualizer. The Gromacs 2018 was used to calculate the potential energy of the dimers [64]. The dimers were solvated with TIP3P water molecules in a cubic box with periodic boundary conditions of $1.0 \mathrm{~nm}$ from the edge of the box [65]. Since the histones are rich in positively charged residues, the overall positive charge of the system was neutralized by adding chlorine as counter ions. The systems were energy minimized by the steepest descent algorithm with the tolerance of maximum force of $1000.0 \mathrm{~kJ} / \mathrm{mol} / \mathrm{nm}$, implementing OPLSAA force field [66]. The potential energies values of the dimers were clustered using the R package 'gplots' to plot the heat-maps of histone $\mathrm{H} 2 \mathrm{~A}$ and $\mathrm{H} 2 \mathrm{~B}$ isoform dimers of human, rat, and mouse.

\section{Key points}

- HISTome2 provides in-depth information on histone proteins (variants or non-canonical and isoforms or canonical), PTMs and modifying enzymes for human, mouse, and rat on a single platform.

- It also provides detailed information on epidrugs for various categories HATi, HDACi, DNMTi, etc. to highlight their importance in the treatment of different diseases.

- Overall, the HISTome2 has significantly increased the extent and diversity of its content and thus assisting in the search and comparative interpretation of the multifactorial parameters in the field of histone biology.

- The database will serve as a useful "knowledge base" for basic researches, clinical scientists, pharmacologists in understanding the histone biology and their potential importance in epidrug discovery through its ability to connect chemistry, biology, and informatics.

- HISTome2 is freely available at http://www.actre c.gov.in/histome2/.

\section{Acknowledgements}

All authors thank BTIS facility at ACTREC for providing infrastructure facilities for updating the database. We would like to thank S Galande, S Khare, F Habib and R Sharma, contributors for the curation of initial version 'Hlstome: The histone Infobase.' All authors are grateful to Dr. Sanjeev Galande for motivational support in updation of the database. The authors are thankful to Mr. Shyam Chavan, Photography section, ACTREC for drawing the graphics displayed on the homepage.

\section{Authors' contributions}

SG and NG conceived the idea of updating and designing the layout of the database. SS, AN, DR, and MR performed data mining and literature search. SS and MR wrote notes for database entries. TM designed the user interface and wrote PHP/HTML code with NG support. PK curated data for EpiDrug and wrote PHP/HTML code. SS, AN, NG, and SG wrote the manuscript. All authors 
critically validated the database, read and approved the final version of the database. All authors read and approved the final manuscript.

\section{Funding}

No funding received for updation of the HIstome database to HISTome2: The HISTone infobase.

\section{Availability of data and materials}

HISTome2 data are available at http://www.actrec.gov.in/histome2/

\section{Ethics approval and consent to participate}

Not applicable.

\section{Consent for publication}

Not applicable.

\section{Conflict of interest}

None to declare.

\begin{abstract}
Author details
${ }^{1}$ Epigenetics and Chromatin Biology Group, Gupta Laboratory, Cancer Research Institute, Advanced Centre for Treatment, Research and Education in Cancer, Tata Memorial Centre, Kharghar, Navi Mumbai, MH 410210, India. 2 Bioinformatics Centre, Advanced Centre for Treatment, Research and Education in Cancer, Tata Memorial Centre, Kharghar, Navi Mumbai, MH 410210, India. ${ }^{3}$ Homi Bhabha National Institute, Training School Complex, Anushakti Nagar, Mumbai, MH 400085, India. ${ }^{4}$ Present Address: Diabetes Unit, King Edward Memorial Hospital Research Centre, Rasta Peth, Pune, Maharashtra 411 011, India. ${ }^{5}$ Present Address: Stowers Institute for Medical Research, Kansas City, MO 64110, USA.
\end{abstract}

Received: 27 May 2020 Accepted: 28 July 2020

Published online: 03 August 2020

\section{References}

1. Mariño-Ramírez L, Kann MG, Shoemaker BA, Landsman D. Histone structure and nucleosome stability. Expert Rev Proteomics. 2005;2:719-29.

2. Wang Y, Xiao J, Suzek TO, Zhang J, Wang J, Zhou Z, et al. PubChem's BioAssay Database. Nucleic Acids Res. 2012;40:D400-12.

3. Chow J, Heard E. X inactivation and the complexities of silencing a sex chromosome. Curr Opin Cell Biol. 2009;21:359-66.

4. Oliver SS, Denu JM. Dynamic interplay between histone H3 modifications and protein interpreters: emerging evidence for a "Histone Language". ChemBioChem. 2011;12:299-307.

5. Bannister AJ, Kouzarides T. Regulation of chromatin by histone modifications. Cell Res. 2011;21:381-95.

6. Buschbeck M, Hake SB. Variants of core histones and their roles in cell fate decisions, development and cancer. Nat Rev Mol Cell Biol [Internet]. Nature Publishing Group; 2017;18:299-314. http://www.nature.com/artic les/nrm.2016.166. Accessed 30 July 2019.

7. Singh R, Harshman SW, Ruppert AS, Mortazavi A, Lucas DM, ThomasAhner JM, et al. Proteomic profiling identifies specific histone species associated with leukemic and cancer cells. Clin Proteomics [Internet]. BioMed Central; 2015;12:22. http://www.pubmedcentral.nih.gov/artic lerender.fcgi?artid=4551702\&tool=pmcentrez\&rendertype=abstract

8. Khan SA. Global histone post-translational modifications and cancer: biomarkers for diagnosis, prognosis and treatment? World J Biol Chem. 2015;6:333.

9. Schones DE, Leung A, Natarajan R. Chromatin modifications associated with diabetes and obesity. Arterioscler Thromb Vasc Biol. 2015;35:1557-61.

10. Araki Y, Mimura T. The histone modification code in the pathogenesis of autoimmune diseases. Mediators Inflamm. 2017. https://doi. org/10.1155/2017/2608605.

11. Sharda A, Amnekar RV, Natu A, Sukanya, Gupta S. Histone posttranslational modifications: potential role in diagnosis, prognosis, and therapeutics of cancer. Progn Epigenetics. Elsevier; 2019. p. 351-73.
12. Sporn JC, Kustatscher G, Hothorn T, Collado M, Serrano M, Muley T, et al. Histone macroH2A isoforms predict the risk of lung cancer recurrence. Oncogene. 2009;28:3423-8.

13. Pericentric heterochromatin becomes enriched with H2A.Z during early mammalian development [Internet]. https://www.ncbi.nlm.nih.gov/pmc/ articles/PMC152904/. Accessed 14 July 2020.

14. Hu G, Cui K, Northrup D, Liu C, Wang C, Tang Q, et al. H2A.Z facilitates access of active and repressive complexes to chromatin in embryonic stem cell self-renewal and differentiation. Cell Stem Cell. 2013;12:180-92.

15. Vardabasso C, Hake SB, Bernstein E. Histone variant H2A.Z.2: A novel driver of melanoma progression. Mol Cell Oncol. 2016;3:1-2. https:// doi.org/10.1080/23723556.2015.1073417.

16. Tomonaga T, Matsushita K, Yamaguchi S, Oohashi T, Shimada H, Ochiai $\mathrm{T}$, et al. Overexpression and mistargeting of centromere protein-A in human primary colorectal cancer. Cancer Res. 2003;63:3511-6.

17. Marmorstein R, Trievel RC. Histone modifying enzymes: structures, mechanisms, and specificities. Biochim Biophys Acta. 2009;1789:58-68.

18. Zhen L, Gui-Lan L, Ping Y, Jin H, Ya-Li W. The expression of H3K9Ac, H3K14AC, and H4K20TriMe in epithelial ovarian tumors and the clinical significance. Int J Gynecol Cancer. 2010;20:82-6.

19. Bianco-Miotto T, Chiam K, Buchanan G, Jindal S, Day TK, Thomas M, et al. Global levels of specific histone modifications and an epigenetic gene signature predict prostate cancer progression and development. Cancer Epidemiol Biomarkers Prev. 2010;19:2611-22.

20. Gao Y, Geng J, Hong X, Qi J, Teng Y, Yang Y, et al. Expression of p300 and CBP is associated with poor prognosis in small cell lung cancer. Int J Clin Exp Pathol 2014;7:760-7. http://www.ijcep.com/. Accessed 14 July 2020.

21. Ishihama K, Yamakawa M, Semba S, Takeda H, Kawata S, Kimura S, et al. Expression of HDAC 1 and CBP/p300 in human colorectal carcinomas. J Clin Pathol [Internet]. BMJ Publishing Group; 2007;60:1205-10. /pmc/ articles/PMC2095491/?report=abstract. Accessed 20 July 2020.

22. Li Y, Seto E. HDACs and HDAC inhibitors in cancer development and therapy. Cold Spring Harb Perspect Med [Internet]. Cold Spring Harbor Laboratory Press; 2016;6. /pmc/articles/PMC5046688/?report=abstract. Accessed 20 July 2020.

23. Gan L, Yang Y, Li Q, Feng Y, Liu T, Guo W. Epigenetic regulation of cancer progression by EZH2: From biological insights to therapeutic potential [Internet]. Biomark. Res. BioMed Central Ltd.; 2018. /pmc/articles/ PMC5845366/?report=abstract. Accessed 20 July 2020.

24. Sethi G, Shanmugam MK, Arfuso F, Kumar AP. Role of RNF20 in cancer development and progression-a comprehensive review [Internet]. Biosci. Rep. Portland Press Ltd; 2018. p. 20171287. /pmc/articles/ PMC6043722/?report = abstract. Accessed 20 July 2020.

25. Cicenas J, Zalyte E, Bairoch A, Gaudet P. Kinases and cancer [Internet]. Cancers (Basel). MDPI AG; 2018. https://www.ncbi.nlm.nih.gov/pmc/ articles/PMC5876638/. Accessed 15 July 2020.

26. Suraweera A, O'Byrne KJ, Richard DJ. Combination therapy with histone deacetylase inhibitors (HDACi) for the treatment of cancer: achieving the full therapeutic potential of HDACi. Front Oncol. 2018;8:92.

27. Zhang Y, Lv J, Liu H, Zhu J, Su J, Wu Q, et al. HHMD: the human histone modification database. Nucleic Acids Res. 2010;38:D149-54.

28. Gendler K, Paulsen T, Napoli C. ChromDB: the chromatin database. Nucleic Acids Res. 2008;36:D298-302.

29. Huang H, Maertens AM, Hyland EM, Dai J, Norris A, Boeke JD, et al. HistoneHits: a database for histone mutations and their phenotypes. Genome Res. 2009;19:674-81.

30. Marino-Ramirez L, Levine KM, Morales M, Zhang S, Moreland RT, Baxevanis AD, et al. The histone database: an integrated resource for histones and histone fold-containing proteins. Database [Internet]. 2011;2011:bar048-bar048. http://www.ncbi.nlm.nih.gov/pubmed/22025 671. Accessed 31 July 2019.

31. Qi Y, Wang D, Wang D, Jin T, Yang L, Wu H, et al. HEDD: the human epigenetic drug database. Database [Internet]. Narnia; 2016;2016:baw159. https://academic.oup.com/database/article-lookup/doi/10.1093/datab ase/baw159. Accessed 4 Sept 2019.

32. Khare SP, Habib F, Sharma R, Gadewal N, Gupta S, Galande S. Hlstome-a relational knowledgebase of human histone proteins and histone modifying enzymes. Nucleic Acids Res [Internet]. Narnia; 2012;40:D337-42. 
https://academic.oup.com/nar/article-lookup/doi/10.1093/nar/gkr1125. Accessed 31 July 2019.

33. FireBrowse.org [Internet]. http://firebrowse.org/. Accessed 4 Sept 2019.

34. Agarwal V, Bell GW, Nam J-W, Bartel DP. Predicting effective microRNA target sites in mammalian mRNAs. Elife [Internet]. 2015;4. https://elife sciences.org/articles/05005. Accessed 6 Aug 2019.

35. Weinstein JN, Collisson EA, Mills GB, Shaw KRM, Ozenberger BA, Ellrott K, et al. The Cancer Genome Atlas Pan-Cancer analysis project. Nat Genet [Internet]. 2013;45:1113-20. http://www.nature.com/articles/ng.2764. Accessed 4 Sept 2019

36. Weininger D. SMILES. 3. DEPICT. Graphical depiction of chemical structures. J Chem Inf Model. 1990;30:237-43. https://doi.org/10.1021/ci000 $67 \mathrm{a} 005$.

37. Weininger $D$, Weininger A, Weininger JL. SMILES. 2. Algorithm for generation of unique SMILES notation. J Chem Inf Model. 1989;29:97-101. https ://doi.org/10.1021/ci00062a008.

38. Weininger D. SMILES, a chemical language and information system. 1 . Introduction to methodology and encoding rules. J Chem Inf Model. 1988;28:31-6. https://doi.org/10.1021/ci00057a005.

39. Heller SR, McNaught A, Pletnev I, Stein S, Tchekhovskoi D. InChl, the IUPAC international chemical identifier. J Cheminform. 2015;7:23.

40. Heller S, McNaught A, Stein S, Tchekhovskoi D, Pletnev I. InChl一-the worldwide chemical structure identifier standard. J Cheminform. 2013;5:7.

41. Frey J, De Roure D, Taylor K, Essex J, Mills H, Zaluska E. CombeChem: a case study in provenance and annotation using the semantic web. Springer, Berlin, Heidelberg; 2006. p. 270-7. http://link.springer. com/10.1007/11890850_27. Accessed 4 Sept 2019.

42. Bento AP, Gaulton A, Hersey A, Bellis LJ, Chambers J, Davies M, et al. The ChEMBL bioactivity database: an update. Nucleic Acids Res. 2014:42:D1083-90.

43. Irwin JJ, Sterling T, Mysinger MM, Bolstad ES, Coleman RG. ZINC: a free tool to discover chemistry for biology. J Chem Inf Model. 2012;52:1757-68.

44. Wishart DS, Feunang YD, Marcu A, Guo AC, Liang K, Vázquez-Fresno R, et al. HMDB 4.0: the human metabolome database for 2018. Nucleic Acids Res. 2018;46:D608-17.

45. Hoofnagle JH, Serrano J, Knoben JE, Navarro VJ. LiverTox: a website on drug-induced liver injury. Hepatology. 2013;57:873-4.

46. Frolkis A, Knox C, Lim E, Jewison T, Law V, Hau DD, et al. SMPDB: the small molecule pathway database. Nucleic Acids Res. 2010;38:D480-7.

47. Jin C, Felsenfeld G. Nucleosome stability mediated by histone variants H3.3 and H2A.Z. Genes Dev. 2007;21:1519-29.

48. Shah S, Verma T, Rashid M, Gadewal N, Gupta S. Histone H2A isoforms : potential implications in epigenome plasticity and diseases in eukaryotes. 2020;0123456789.

49. Bhattacharya S, Reddy D, Jani V, Gadewal N, Shah S, Reddy R, Bose K, Sonavane U, Joshi R, Smoot D, Ashktorab H, Gupta S. Histone isoform $\mathrm{H} 2 \mathrm{~A} 1 \mathrm{H}$ promotes attainment of distinct physiological states by altering chromatin dynamics. Epigenetics Chromatin. 2017;10:48.

50. Sievers F, Higgins DG. Clustal omega, accurate alignment of very large numbers of sequences. Methods Mol Biol [Internet]. 2014. p. 105-16. http://www.ncbi.nlm.nih.gov/pubmed/24170397. Accessed 8 Aug 2019.

51. Crooks GE, Hon G, Chandonia J-M, Brenner SE. WebLogo: a sequence logo generator. Genome Res. 2004;14:1188-90.

52. Stelzer G, Rosen N, Plaschkes I, Zimmerman S, Twik M, Fishilevich S, et al. The genecards suite: from gene data mining to disease genome sequence analyses. Curr Protoc Bioinforma [Internet]. Hoboken, NJ, USA: John Wiley \& Sons, Inc;; 2016. p. 1.30.1-1.30.33. http://doi.wiley .com/10.1002/cpbi.5. Accessed 4 Sept 2019.
53. Geer LY, Marchler-Bauer A, Geer RC, Han L, He J, He S, et al. The NCBI BioSystems database. Nucleic Acids Res. 2010;38:D492-6.

54. UniProt: a worldwide hub of protein knowledge. Nucleic Acids Res [Internet]. Narnia; 2019;47:D506-15. https://academic.oup.com/nar/article/47/ D1/D506/5160987. Accessed 4 Sept 2019.

55. Draizen EJ, Shaytan AK, Mariño-Ramírez L, Talbert PB, Landsman D, Panchenko AR. HistoneDB 2.0: a histone database with variants - an integrated resource to explore histones and their variants. Database. 2016;2016:1-10.

56. Talbert PB, Ahmad K, Almouzni G, Ausiá J, Berger F, Bhalla PL, et al. A unified phylogeny-based nomenclature for histone variants. Epigenetics Chromatin. 2012;5:1-19.

57. Bruford EA, Lush MJ, Wright MW, Sneddon TP, Povey S, Birney E. The HGNC Database in 2008: a resource for the human genome. Nucleic Acids Res [Internet]. Narnia; 2007;36:D445-8. https://academic.oup.com/nar/artic le-lookup/doi/10.1093/nar/gkm881. Accessed 6 Aug 2019.

58. Shimoyama M, De Pons J, Hayman GT, Laulederkind SJF, Liu W, Nigam R, et al. The Rat Genome Database 2015: genomic, phenotypic and environmental variations and disease. Nucleic Acids Res. 2015;43:D743-50.

59. Bult CJ, Blake JA, Smith CL, Kadin JA, Richardson JE, Anagnostopoulos A, et al. Mouse Genome Database (MGD) 2019. Nucleic Acids Res. 2019:47:D801-6.

60. Burley SK, Berman HM, Bhikadiya C, Bi C, Chen L, Di Costanzo L, et al. RCSB Protein Data Bank: biological macromolecular structures enabling research and education in fundamental biology, biomedicine, biotechnology and energy. Nucleic Acids Res [Internet]. Narnia; 2019;47:D464-74. https://academic.oup.com/nar/article/47/D1/D464/5144139. Accessed 4 Sept 2019.

61. Dreos R, Ambrosini G, Périer RC, Bucher P. The Eukaryotic Promoter Database: expansion of EPDnew and new promoter analysis tools. Nucleic Acids Res [Internet]. Narnia; 2015;43:D92-6. http://academic.oup.com/ nar/article/43/D1/D92/2437610/The-Eukaryotic-Promoter-Databaseexpansion-of. Accessed 4 Sept 2019.

62. Kim S, Thiessen PA, Bolton EE, Chen J, Fu G, Gindulyte A, et al. PubChem substance and compound databases. Nucleic Acids Res. 2016:44:D1202-13.

63. https://clinicaltrials.gov/[Internet]. https://clinicaltrials.gov/. Accessed 4 Sept 2019.

64. Pronk S, Páll S, Schulz R, Larsson P, Bjelkmar P, Apostolov R, et al. GROMACS 4.5: a high-throughput and highly parallel open source molecular simulation toolkit. Bioinformatics [Internet]. Narnia; 2013;29:845-54. https://academic.oup.com/bioinformatics/article-lookup/doi/10.1093/ bioinformatics/btt055. Accessed 8 Aug 2019.

65. Jorgensen WL, Chandrasekhar J, Madura JD, Impey RW, Klein ML. Comparison of simple potential functions for simulating liquid water. J Chem Phys. 1983;79:926-35. https://doi.org/10.1063/1.445869.

66. Jorgensen WL, Tirado-Rives J. The OPLS [optimized potentials for liquid simulations] potential functions for proteins, energy minimizations for crystals of cyclic peptides and crambin. J Am Chem Soc. 1988;1 10:165766. https://doi.org/10.1021/ja00214a001.

\section{Publisher's Note}

Springer Nature remains neutral with regard to jurisdictional claims in published maps and institutional affiliations. 